PAPER

\title{
Psychosocial impact of finding small aneurysms that are left untreated in patients previously operated on for ruptured aneurysms
}

\author{
I C van der Schaaf, M J H Wermer, B K Velthuis, E Buskens, P M M Bossuyt, G J E Rinkel
}

See Editorial Commentary, p 713

J Neurol Neurosurg Psychiatry 2006;77:748-752. doi: 10.1136/jnnp.2005.079194

See end of article for authors' affiliations

Correspondence to

Correspondence to:
Irene $\mathrm{C}$ van der Schaaf

Department of Radiology

E01.132, University

Medical Centre Utrecht

PO Box 85500, 3508 GA

Utrecht, the Netherlands:

i.vanderschaał@rrr.azu.nl

Received 26 August 2005 In revised form

28 December 2005

Accepted 5 January 2006
Objectives: In patients with previous subarachnoid haemorrhage (SAH) undergoing follow up screening, the authors assessed the impact of finding but not treating very small aneurysms by comparing quality of life (QOL), anxiety, and depression between patients with a newly detected aneurysm that was left untreated (cases) and patients with a negative screening (controls) as this should be incorporated in the evaluation of effectiveness of screening.

Methods: In patients with previous SAH undergoing screening for new aneurysms the authors compared QOL (SF-36, EURO-QOL, and a screening related questionnaire), anxiety, and depression (Hospital Anxiety and Depression Scale (HADS)) between cases and controls. Differences in scores on the SF-36, EURO-QOL, and HADS were assessed with Student's $t$ test and differences in proportions of patients with HADS scores in the pathological range and screening related changes with $\chi^{2}$ analysis. The authors powered the study to detect a moderate, clinically relevant difference.

Results: Thirty five cases and 34 controls were included. Trends for health related $Q O L$, anxiety, depression, and consequences in daily life pointed in the same direction of a less favourable situation for cases but all effects were small, and did not reach statistical significance. On the screenings specific questionnaire, cases more often (but not statistically significant) reported changes in daily life.

Conclusions: The authors found no major or moderate impact on QOL, anxiety, and depression of the awareness of having an untreated aneurysm, which was detected at screening, although most items showed a trend towards more negative effects for cases. Minor effects on individual level cannot be excluded by this study.
I ntracranial aneurysms have long been considered a once in a lifetime event. Nevertheless, patients who survive after subarachnoid haemorrhage (SAH) may be at risk of developing new episodes of SAH from regrowth aneurysms at the clip site or new aneurysms at other sites. ${ }^{1-4}$ Second episodes of SAH from new aneurysms also carry a poor prognosis. ${ }^{5}$ Therefore, screening for new aneurysms may be beneficial. Before screening is implemented in clinical practice, the cost effectiveness should be evaluated. We performed a screenings study for this purpose.

Very small aneurysms, found with screening, are often not treated because the risk of treating these small aneurysms does not outweigh the potential benefit. ${ }^{6}$ The increasing resolution of multislice CTA (MS-CTA) has been paralleled by a growing number of patients with a very small aneurysm detected at screening without immediate consequences for treatment.

An evaluation of the effectiveness of screening should take into account both the risks of rupture and treatment complications as well as the psychosocial impact of detecting, but not treating an aneurysm. To evaluate this effect, patients with an untreated aneurysm detected by screening could be compared with screened patients in whom no aneurysm is found. In both patient groups the quality of life will be similarly affected by the history of $\mathrm{SAH}^{7}$ and by the performance of screening. ${ }^{8}$ The objective of this study is to assess the impact of finding at screening very small aneurysms that are not treated in a series of patients with a previous episode of SAH who underwent screening for de novo aneurysms. We therefore compared the health related quality of life and symptoms of anxiety and depression between patients with a newly detected small aneurysm that was left untreated and patients with a negative screening result.

\section{METHODS \\ Patients}

For the present study, participants were recruited from a study in which the (cost) effectiveness of screening for new aneurysms was studied in patients clipped for ruptured intracranial aneurysms (the ASTRA study ${ }^{9}$ ). Patients were eligible for screening if they were between 20 and 70 years of age and able to care for themselves in daily life. Patients with serious comorbidity decreasing expected years of life, or increasing complication risk of treatment, were excluded. The study was approved by the medical ethics committees of the two participating university hospitals.

The case group consisted of patients with a small aneurysm detected by screening that was left untreated. The control group consisted of patients in the same study but no aneurysm detected on screening. Control patients were matched firstly on age, secondly on sex, and thirdly, if possible on the time interval between screening and QOL assessment

\section{Instruments and patients characteristics}

We assessed quality of life by means of the validated Dutch versions of the MOS Short Form (SF-36) and the EURO QOL (EQ-5D) including a visual analogue scale (EQ-VAS). ${ }^{10}{ }^{11}$ We selected these instruments because of their psychometric qualities and for practical reasons. The SF-36 contains 36 questions covering eight health related domains: physical functioning, role limitations because of physical health problems, bodily pain, social functioning, general mental

Abbreviations: HADS, Hospital Anxiety and Depression Scale; QOL, quality of life; SAH, subarachnoid haemorrhage. 
health, role limitations because of emotional problems, vitality, and general health perception. Higher scores indicate a better QOL. ${ }^{10}{ }^{12}$ The EQ-5D comprises five questions addressing mobility, daily activities, pain, self care, and anxiety and depression. Responses are converted to a best score of 1 and worst score of 0 . On the visual analog scale, the overall health can be rated on a scale of 0 (worst) to 100 (best). ${ }^{11}$ To determine states of anxiety and depression we administered the Hospital Anxiety and Depression scale (HADS). On the basis of 14 questions on mood, severity of anxiety and depression was categorised into "normal" (scores <8), doubtful (scores 8-10), and scores in the pathological range (scores $>10$ ). ${ }^{13-15}$ In addition we administered a screening specific questionnaire that was developed to measure changes and consequences in daily life related to screening for intracranial aneurysms. This instrument contained items on changes in lifestyle (diet, exercise habits, medication, smoking, and alcohol), social circumstances and emotional wellbeing (level of independence, self esteem, relationship, coping with stress, future outlook), fear about daily activities, insurance, driver's license, and work, which all may have been affected by screening. Patients were also asked specifically if they regretted undergoing the screening test. All instruments were self administered by the respondents.

We collected information on age, sex, time after screening and after SAH, handicap, the number of patients with a second SAH or with an ischaemic stroke or transient ischaemic attack (TIA) during the period of follow up after the initial SAH, dysphasia, focal deficits, cognitive impairment, and educational and cultural background. Handicap was assessed by means of the modified Rankin scale ${ }^{16}$ Educational background was categorised as: primary school or less, secondary school or vocational training, and higher education or university. Cultural background was defined as a white and non-white background.

\section{Data collection}

Patients were approached approximately six months after screening. Patients with small aneurysms that remain untreated are screened again one year after the initial screening to obtain information on growth of the aneurysm. The time window of half a year was chosen to reduce the chance of measuring the influence of tension from the initial screening or follow up screening on QOL. Cases and controls were contacted by telephone by one of the researchers (IS) to give information on the study purpose and the questionnaires, and to ask for participation. If patients agreed to participate an information letter together with the questionnaires were sent to the patients.

\section{Data analysis}

SF-36 domain scores were calculated according to standard guidelines, and analysed using standard parametric methods. ${ }^{12}$ Differences between cases and controls in scores on the SF-36, the EURO-QOL were assessed with the Student's $t$ test. Also for the HADS, differences in anxiety and depression subscale scores were compared with the Student's $t$ test. Since in samples with minor psychiatric disorders symptoms of anxiety and depression covary to a considerable extent, ${ }^{14}$ we performed an additional analysis combining anxiety and depression scores (total scale). The difference in proportions of cases and controls with definite anxiety or depression and differences between cases and controls for the screenings specific questionnaire were tested by means of the chi-squared test. All estimates were reported with 95\% confidence intervals (95\% CI).

The sample size calculation of the patients group was based upon the standardised difference in the general health state subscale of the SF-36. ${ }^{17}$ This is the ratio of the difference of interest to the standard deviation of the observations. The standard deviation of this health state was derived from the control group. Based upon a power of $80 \%$ and a significance level of 0.05 the minimal size of the patients group had to be 26 to detect a moderate, clinically relevant difference. ${ }^{18}$

\section{RESULTS}

\section{Patients}

Thirty nine patients with an aneurysm detected by screening which was left untreated were contacted, of which one declined to participate and three did not respond despite a second request. Thirty seven patients without a new aneurysm on screening were contacted. Three did not respond despite a second request; all other patients agreed to participate. Eventually we were able to include 35 cases with an untreated aneurysm (response rate 90\%) and 34 controls without an aneurysm (92\%). Patient characteristics in both groups were balanced except for period of follow up after SAH, which was 9.1 years in the cases group and 7.1 years in the control group. For the additional QOL related patient characteristics, information was available for all but one of the cases and controls. QOL related patient characteristics were comparable in both groups, except for educational background which was lower for cases and for the Rankin score which was somewhat less favourable for cases (table 1).

\section{Quality of life}

Figure 1 shows the mean SF-36 scores of the cases and control group. The SF-36 scores of the cases were lower for all subdomains, most predominant for physical functioning, bodily pain, general health, and role emotional. None of these differences was statistically significant.

The mean score of the EQ-5D was 0.8 in the cases group and 0.9 in the control group (difference of means $0.07 ; 95 \%$ CI -0.01 to 0.15$)$. On the EQ-VAS, cases assessed their overall health state lower than the controls. The mean score for the cases was 75.3 against 81.1 for the controls (difference of means $5.8 ; 95 \% \mathrm{CI}-1.0$ to 12.6 ).

Table 1 Patient characteristics and additional QOL related parameters

\begin{tabular}{lll}
\hline & & \multicolumn{1}{c}{ Controls } \\
& Cases (n=35) & $(\mathbf{n}=34)$ \\
\hline Mean age (range) (years) & $55.2(31-70)$ & $55.5(29-67)$ \\
Men (\%) & $10(29 \%)$ & $13(38 \%)$ \\
Mean interval after screening (months) & 6.4 & 6.9 \\
Mean interval since SAH (years) & 9.1 & 7.1 \\
QOL related parameters & $n=34$ & $n=33$ \\
Rankin score & (percentage) & \\
0 & $15(44 \%)$ & $18(55 \%)$ \\
1 & $10(29 \%)$ & $10(30 \%)$ \\
2 & $7(21 \%)$ & $4(12 \%)$ \\
3 & $2(5.8 \%)$ & $1(3 \%)$ \\
Repeated SAH & $1(3 \%)$ & $0(0 \%)$ \\
Ischaemic stroke/TIA* & $3(8.8 \%)$ & $3(9.1 \%)$ \\
Dysphasia & $0(0 \%)$ & $2(6.1 \%)$ \\
Focal deficits & $5(15 \%)$ & $3(9.1 \%)$ \\
Cognitive impairment (mild) & $22(65 \%)$ & $24(73 \%)$ \\
Educational background & $18(53 \%)$ & $11(33 \%)$ \\
Primary school or less & $10(29 \%)$ & $15(46 \%)$ \\
Secondary school/vocational & & \\
training & $6(18 \%)$ & $7(21 \%)$ \\
Higher education or university & & 34 \\
Cultural background & 33 & $0(0 \%)$ \\
White & $1(3 \%)$ & \\
Non-white & & \\
\hline & & \\
\hline
\end{tabular}




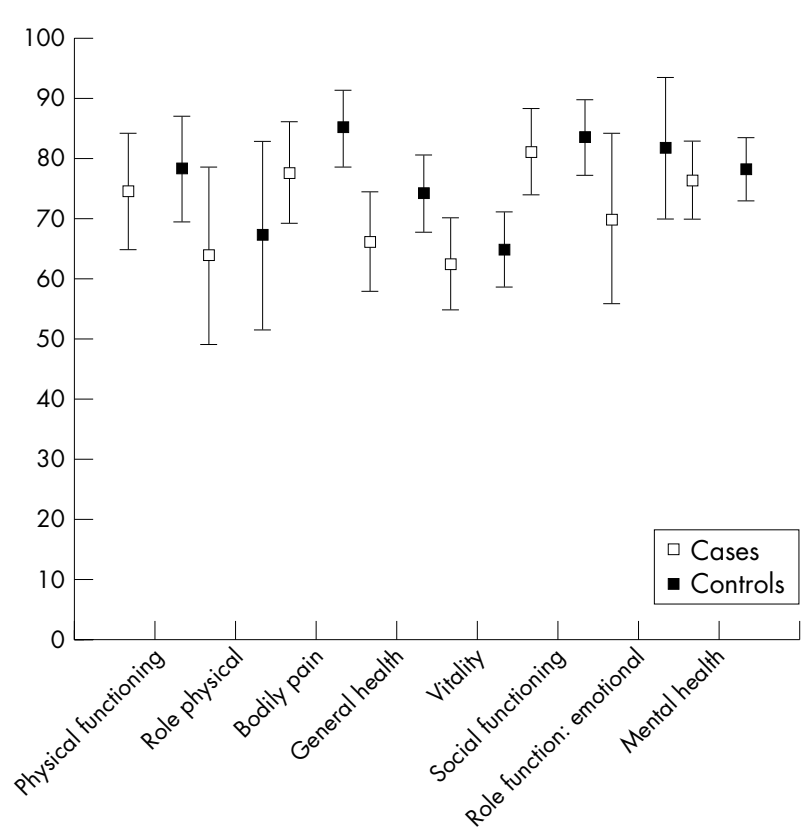

Figure 1 Mean SF-36 domain scores for patients and controls. Means and corresponding $95 \%$ confidence intervals in the cases and controls for each subdomain of the SF-36.

\section{States of anxiety and depression}

The mean subscore for anxiety was 5.4 (SD 3.6) for the cases and 3.9 (SD 2.9) for the controls; (difference of means 1.6; $95 \%$ CI -0.04 to 3.2 ). Four of the 35 cases had a score for anxiety in the pathological range compared to none of the 34 controls, a difference of $11 \%$; $95 \%$ CI $-0.8 \%$ to $26 \%$. The mean subscore for depression was 5.9 (SD 3.1) in the cases and 5.7 (SD 2.7) in the control group (difference of means $0.2 ; 95 \% \mathrm{CI}-1.2$ to 1.7 ). For the depression subscore, four cases had a score in the pathological range compared to one patient in the control group, a difference of $8.5 \%$; $95 \%$ CI $-5.3 \%$ to $23 \%$ (fig 2 ). The mean total scale score was 11.4 (SD 5.6) for the cases and 9.6 (SD 5.0) for the controls; (difference of means $1.8 ; 95 \%$ CI -0.8 to 4.4 ).

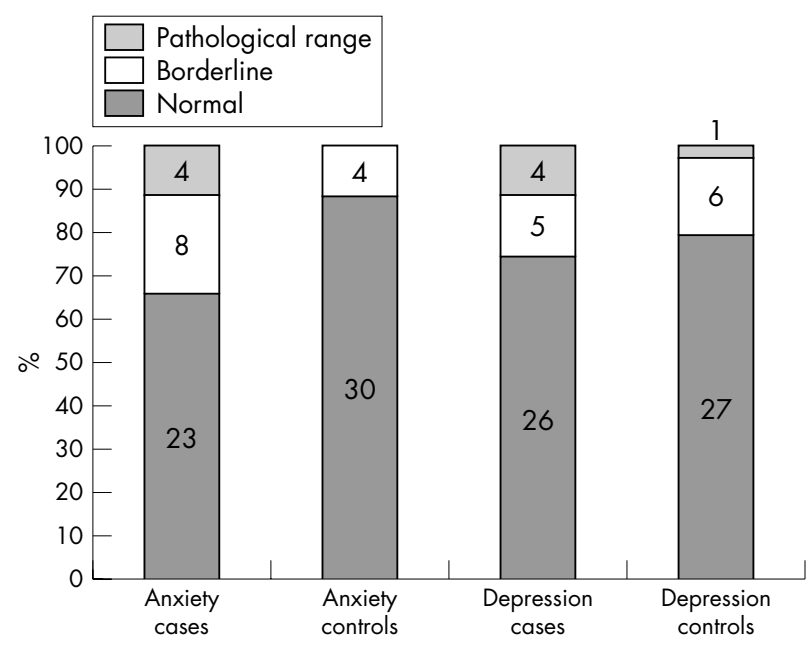

Figure 2 Categorisation of subscores for anxiety and depression in cases and controls. Number of cases and controls with definite, borderline, and normal levels of anxiety and depression are shown in the bars.
Changes and consequences of screening in daily life

Table 2 presents the responses of the cases and controls on questions about changes in lifestyle, socioemotional behaviour, fears in daily activities, insurance, driving license, and work.

There were 28 (80\%) cases who had changed to a healthier lifestyle, compared to 20 (59\%) controls (difference $21 \%$; $95 \%$ CI $-1 \%$ to $40 \%$ ). Negative changes in social circumstances or emotional wellbeing were reported by $15(43 \%)$ cases and 13 (38\%) controls (difference $5 \%$; $95 \%$ CI $-18 \%$ to $26 \%$ ). Eleven $(31 \%)$ cases and six $(18 \%)$ controls reported more fear in daily activities (difference 14\%; $95 \%$ CI $-7 \%$ to $33 \%$ ). There were five cases (14\%) who were denied or faced with increased payment for insurance compared with two controls (6\%) (difference $8 \% ; 95 \%$ CI $-9 \%$ to $25 \%$ ). None of the cases and one of the controls lost their driving license (difference $-3 \%$; $95 \%$ CI $-6 \%$ to $12 \%$ ). Three cases (9\%) had stopped working or were working fewer hours compared with five controls (15\%) (difference 6\%; $95 \%$ CI $-10 \%$ to $23 \%$ ). In the cases group, two of the three patients stopped working voluntary and one patient became unemployed due to the screening. Of the five controls who stopped working, two did so voluntary and the other three for reasons other than the screening. Five of the cases (14\%) and one of the controls (3\%) regretted participation in the screening programme (difference $11 \%$; $95 \%$ CI $-3 \%$ to $27 \%$ ).

\section{DISCUSSION}

We found no major or moderate, clinically relevant differences on health related QOL and mood between patients with a new, small aneurysm detected at screening that is left untreated and patients with a negative screening result. Therefore, in the evaluation of the effectiveness of screening, aspects of QOL and anxiety and depression do not play a substantial role.

Although scores on the SF-36 and the EQ-5D were lower for the cases, the differences were not statistically significant. Both are generic instruments. The SF-36 has been recommended for use in QOL studies on vascular disease; ${ }^{19}$ however, an important concern with a generic instrument is that it may be unresponsive to effects specifically related to this study population. Therefore we also applied an instrument constructed specifically for patients screened on intracranial aneurysms. With regard to our assessment on the effect of screening on daily life, we found a trend towards more negative effects on social circumstances, emotional wellbeing, and fear in daily activities in cases. More than three quarters of cases and more than half of controls reported that they had changed their lifestyle to healthier living. Although this finding can be attributed by concerns about a healthy lifestyle that increase with age, or are becoming more prevalent in society, it could also be read as signs of fear induced by the awareness that a new aneurysm may develop and by the screening procedure itself. Also, patients with an untreated aneurysm more often regretted participation, but this difference was not statistically significant.

This study was powered to detect moderate clinically significant differences. We cannot draw conclusions on small differences in QOL, anxiety, and depression. We considered the detection of smaller differences with sufficient precision not to be clinically relevant; moreover for such detection a larger number of patients would be required. In the evaluation of the effectiveness of screening, small effects in a relatively small proportion of the screened population do not have a considerable impact. On individual basis these effects, especially symptoms of anxiety, may play an important role and therefore attention should be paid to the psychosocial status of patients with a positive screening. 
Table 2 Screenings specific questionnaire smoked before screening

\begin{tabular}{|c|c|c|c|c|c|}
\hline \multirow[b]{2}{*}{ Changes in lifestyle } & \multicolumn{2}{|c|}{ Cases $(n=35)$} & \multicolumn{2}{|c|}{ Controls $(n=34$ ) } & \multirow{2}{*}{$\begin{array}{l}\text { Difference }(95 \% \mathrm{Cl}) \\
21 \%(-0.5 \% \text { to } 40 \%)\end{array}$} \\
\hline & 28 & $80 \%$ & 20 & $59 \%$ & \\
\hline Diet (cutting down fat, salt, quantity) & 17 & $49 \%$ & 11 & $32 \%$ & $16 \%(-9.5 \%$ to $42 \%)$ \\
\hline More exercise & 10 & $29 \%$ & 7 & $21 \%$ & $8 \%(-15 \%$ to $31 \%)$ \\
\hline Medication (start after screening) & 4 & $11 \%$ & 3 & $8.8 \%$ & $2.6 \%(-15 \%$ to $20 \%)$ \\
\hline Smoking (stopped or less) & 13 & $62 \%{ }^{*}$ & 9 & $64 \%{ }^{*}$ & $2.4 \%(-29 \%$ to $31 \%)$ \\
\hline Alcohol (stopped or less) & 3 & $13 \% \dagger$ & 2 & $12 \% \dagger$ & $1.3 \%(-23 \%$ to $22 \%)$ \\
\hline Socioemotional changes & 15 & $43 \%$ & 13 & $38 \%$ & $4.6 \%(-18 \%$ to $26 \%)$ \\
\hline Level of independence (decrease) & 11 & $31 \%$ & 4 & $12 \%$ & $20 \%(-2.1 \%$ to $41 \%)$ \\
\hline Self esteem (decrease) & 11 & $31 \%$ & 7 & $21 \%$ & $11 \%(-13 \%$ to $34 \%)$ \\
\hline Relationship (negative influence) & 1 & $2.9 \%$ & 2 & $5.9 \%$ & $9.5 \%(-9.5 \%$ to $16 \%)$ \\
\hline Coping with stress (worse) & 10 & $29 \%$ & 8 & $24 \%$ & $5 \%(-19 \%$ to $29 \%)$ \\
\hline Future outlook (worse) & 8 & $23 \%$ & 4 & $11 \%$ & $11 \%(-8.9 \%$ to $32 \%)$ \\
\hline Fear in daily activities & 11 & $31 \%$ & 6 & $18 \%$ & $14 \%(-6.7 \%$ to $33 \%)$ \\
\hline Intensive exercise & 4 & $11 \%$ & 2 & $5.9 \%$ & $5.6 \%(-11 \%$ to $22 \%)$ \\
\hline Sexual intercourse & 5 & $14 \%$ & 3 & $8.8 \%$ & $5.5 \%(-13 \%$ to $23 \%)$ \\
\hline Driving a car & 3 & $8.6 \%$ & 2 & $5.9 \%$ & $2.7 \%(-12 \%$ to $18 \%)$ \\
\hline Flying in an airplane & 3 & $8.6 \%$ & 3 & $8.8 \%$ & $0.3 \%(-16 \%$ to $16 \%)$ \\
\hline \multicolumn{6}{|l|}{ Insurance } \\
\hline Senied insurance/increased payment & 5 & $14 \%$ & 2 & $5.9 \%$ & $8.4 \%(-8.5 \%$ to $25 \%)$ \\
\hline \multicolumn{6}{|l|}{ Driving license } \\
\hline Loss or restriction & 0 & $0 \%$ & 1 & $2.9 \%$ & $2.9 \%(-5.6 \%$ to $12 \%)$ \\
\hline \multicolumn{6}{|l|}{ Work } \\
\hline Stopped or working fewer hours & 3 & $8.6 \%$ & 5 & $15 \%$ & $6.1 \%(-10 \%$ to $22.5 \%)$ \\
\hline Regret of participation & 5 & $14 \%$ & 1 & $2.9 \%$ & $11 \%(-3.1 \%$ to $27 \%)$ \\
\hline
\end{tabular}

To facilitate psychosocial help to patients harmed by bearing an untreated small aneurysm detected at screening, information on determinants predisposing for a reduction in QOL would be valuable. Such factors may include age and sex, clinical score, second periods of SAH or ischaemic stroke events, sequelae from previous bleedings and operations (dysphasia, focal deficits, cognitive impairment), and educational and cultural background. We could not perform an analysis to determine predisposing determinants in our patient group as the relatively small number of events does not allow this analysis. However, these factors were balanced in both groups, except for educational background and the Rankin score. Cases had a lower educational background. Since educational background is related to socioeconomic status and income, this may have introduced a bias toward more reduced QOL in cases, because QOL is related to income, even in patients who have had a vascular event. ${ }^{20}$ Cases also had more often restrictions on lifestyle or needed some help in activities of daily life than controls. If this difference in handicap influences the results, this may again have introduced a bias toward more impact on QOL in the cases. Thus, the tendency for more impact on QOL in the cases may very well have been introduced by the difference in baseline characteristics.

In our case group several factors can play a role in influencing QOL. Firstly, QOL might be influenced by the history of SAH. The QOL of patients four and 18 months after $\mathrm{SAH}$ is considerably decreased, except for patients without residual symptoms. ${ }^{7}$ The influence of SAH on long term QOL is unknown. Secondly, the offer of screening itself and the awareness of the chance of developing a new aneurysm may influence QOL and can lead to raised anxiety in both cases and controls. ${ }^{21}{ }^{22}$ To correct for differences in QOL as a result of history of SAH, the effect of screening itself and the impact on patients of awareness that a new aneurysm might develop, we chose the control group from the same screenings population as the cases. From a conceptual point of view it would have been better to compare QOL and anxiety and depression within individuals. For such a comparison we should have sent questionnaires on QOL, anxiety, and depression, and also a screening specific questionnaire to patients years after their SAH without telling that screening would be offered in a later stage (since the offer of screening itself and the awareness that a new aneurysm might develop may influence QOL). Thus, assessments would have had to be done without informing patients why we wanted to have such assessments. From an ethical and pragmatic point of view we found such a design too difficult to conduct. If we had the assessments done after telling the patients that they carried a risk of having an aneurysm, but before screening, the results would have been considerably influenced by the fear induced by telling them that a new aneurysm might develop.

The current study was performed in a series of patients with a history of SAH. Some of these patients will have had neuropsychological deficits from the SAH. These neuropsychological consequences of the SAH might have masked psychosocial consequences of finding but not treating aneurysms. The results of the current study can therefore not be extrapolated to screening for aneurysms in generalthat is, to patients without previous SAH.

The purpose of this study was not to measure the effects of screening but the effect of bearing an untreated aneurysm in a screened population. Measurements were performed half a year after screening to reduce the chance of measuring transient psychological stress from the initial screening or upcoming screening invitation. Comparison with the literature is difficult because of differences in study goals and therefore different methods (patient populations, timing of QOL measurement, and instruments for measuring QOL). In the Multicentre Aneurysm Screening Study, 22 QOL was impaired in the short term after diagnosis (six weeks after screening) but on the long term (three and 12 months) no effects of the diagnosis could be measured. These long term effects are in concordance with our findings. In a study on QOL of patients with an untreated, unruptured intracranial aneurysm but without a previous episode of SAH, patients reported an impaired QOL (measured by the SF-36 and SIP), most prominently in psychosocial domains, without raised anxiety or depression levels (measured by HADS). ${ }^{23}$ The difference between this study and ours can probably be explained by the differences in cases and controls. In the present study both cases and controls had a history of SAH, whereas in the other study the cases were patients without a 
history of SAH but with an untreated aneurysm, while the controls a sample of the Dutch population. The effects on QOL from the previous SAH and the awareness that a new aneurysm might develop, even in those who currently have a negative screening result, may have diluted the contrast between cases and controls in the present study. Cases in our study all had very small aneurysms. They had been told that the aneurysms had a very small risk of rupture and that treatment of the aneurysm would not outweigh the complication risk of treatment. The reassurance we tried to give may have contributed to the negligible effect on QOL, anxiety, and depression in the patients with untreated aneurysms.

One of the contacted cases did not want to participate, three did not respond, and three patients from the control group did not respond. Their motives may be associated with their QOL. Yet we do not think this has biased our results as the participation rate was very high in both groups. This study was performed in the Netherlands. We assume that our population is comparable with other western populations. It is unclear whether results can be extrapolated to countries with a different cultural background.

Currently, screening patients clipped for ruptured intracranial aneurysms should not be advocated. An extensive decision analysis in which all benefits and risks (including the consequences of finding but not treating small aneurysms) of screening are weighed is needed to assess its (cost) effectiveness before screening after $\mathrm{SAH}$ is implemented in clinical practice.

Finding an aneurysm in screening for new aneurysms years after SAH, but not treating this aneurysm has no major or moderate impact on QOL, mood, and psychosocial functioning on group level; however, minor effects on individual level cannot be excluded by our study. Therefore, extra attention should be paid to the psychosocial status of these patients.

\section{Authors' affiliations}

I C van der Schaaf, B K Velthuis, Department of Radiology, University Medical Centre Utrecht, the Netherlands

M J H Wermer, G J E Rinkel, From the Department of Neurology, University Medical Centre Utrecht, the Netherlands

E Buskens, Julius Centre for General Practice and Patient Oriented Research, University Medical Centre Utrecht, the Netherlands

P M M Bossuyt, Department of Clinical Epidemiology and Biostatistics, Academic Medical Centre Amsterdam, the Netherlands

\section{Competing interests: None.}

Ethics approval: This study was approved by the medical ethics committee of the University Medical Centre Utrecht.

\section{REFERENCES}

1 David CA, Vishteh AG, Spetzler RF, et al. Late angiographic follow-up review of surgically treated aneurysms. J Neurosurg 1999;91:396-401.

2 Tsutsumi K, Ueki K, Usui M, et al. Risk of subarachnoid hemorrhage after surgical treatment of unruptured cerebral aneurysms. Stroke 1999;30:1181-4.

3 Tsutsumi K, Ueki K, Morita A, et al. Risk of aneurysm recurrence in patients with clipped cerebral aneurysms: results of long-term follow-up angiography. Stroke 2001;32:1191-4.

4 Juvela S. Risk of subarachnoid hemorrhage from a de novo aneurysm. Stroke 2001;32:1933-4.

5 Wermer MJH, Rinkel GJE, Greebe P, et al. Recurrence of subarachnoid hemorrhage after treatment for ruptured aneurysms: patient characteristics and outcome. Neurosurgery 2005;56:197-204.

6 Wiebers DO, Whisnant JP, Huston J 3rd, et al. Unruptured intracranial aneurysms: natural history, clinical outcome, and risks of surgical and endovascular treatment. Lancet 2003;362:103-10.

7 Hop JW, Rinkel GJE, Algra A, et al. Changes in functional outcome and quality of life in patients and caregivers after aneurysmal subarachnoid hemorrhage. J Neurosurg 2001;95:957-63.

8 Wanhainen A, Rosen C, Rutegard J, et al. Low quality of life prior to screening for abdominal aortic aneurysm: a possible risk factor for negative mental effects. Ann Vasc Surg 2004; 18:287-93.

9 Wermer MJ, van der Schaaf IC, Velthuis BK, et al. Follow-up screening after subarachnoid haemorrhage: frequency and determinants of new aneurysms and enlargement of existing aneurysms. Brain 2005;128:2421-9.

10 Van der Zee KI, Sanderman R, Heyink J. De psychometrische kwaliteiten van de MOS 36- item Short Form Health survey (SF-36) in een Nederlandse populatie. Tijdschr Sociale Gezondheidszorg 1993;71:183-91.

11 Brooks R. EuroQol: the current state of play. Health Policy 1996;37:53-72.

12 McHorney CA, Ware JE Jr, Raczek AE. The MOS 36-Item Short-Form Health Survey (SF-36): II. Psychometric and clinical tests of validity in measuring physical and mental health constructs. Med Care 1993;31:247-63.

13 Zigmond AS, Snaith RP. The hospital anxiety and depression scale. Acta Psychiatr Scand 1983;67:361-70.

14 Spinhoven P, Ormel J, Sloekers PP, et al. A validation study of the Hospital Anxiety and Depression Scale (HADS) in different groups of Dutch subjects. Psychol Med 1997;27:363-70.

15 Snaith RP, Baugh SJ, Clayden AD, et al. The Clinical Anxiety Scale: an instrument derived from the Hamilton Anxiety Scale. Br J Psychiatry 1982;141:518-23.

16 Bamford JM, Sandercock PAG, Warlow CP, et al. Interobserver agreement for the assessment of handicap in stroke patients. Stroke $1989 ; 20: 828$.

17 Wyrwich KW, Fihn SD, Tierney WM, et al. Clinically important changes in health-related quality of life for patients with chronic obstructive pulmonary disease: an expert consensus panel report. J Gen Intern Med 2003; 18:196-202

18 Altman D. Practical statistic for medical research. London, United Kingdom: Chapman \& Hall, 1991:456.

19 Beattie DK, Golledge J, Greenhalgh RM, et al. Quality of life assessment in vascular disease: towards a consensus. Eur J Vasc Endovasc Surg 1997;13:9-13.

20 Visser MC. Measurement of quality of life in patients with ischemic disease of the heart or brain [thesis]. Rotterdam, Netherlands: Erasmus University of Rotterdam, 1996.

21 Lucarotti ME, Heather BP, Shaw E, et al. Psychological morbidity associated with abdominal aortic aneurysm screening. Eur J Vasc Endovasc Surg 1997; 14:499-501.

22 Ashton HA, Buxton MJ, Day NE, et al. The Multicentre Aneurysm Screening Study (MASS) into the effect of abdominal aortic aneurysm screening on mortality in men: a randomised controlled trial. Lancet 2002;360:1531-9.

23 van der Schaaf IC, Brilstra EH, Rinkel GJ, et al. Quality of life, anxiety, and depression in patients with an untreated intracranial aneurysm or arteriovenous malformation. Stroke 2002;33:440-3. 